\title{
PRIME IDEALS IN TWO-DIMENSIONAL POLYNOMIAL RINGS
}

\author{
WILLIAM HEINZER AND SYLVIA WIEGAND
}

(Communicated by Louis J. Ratliff, Jr.)

\begin{abstract}
We show that for every nonzero prime ideal $P$ in a Noetherian domain $R$ there are either just one or infinitely many prime ideals of the absolute integral closure of $R$ lying over $P$. Using this result we show that if $R$ is a semilocal countable one-dimensional Noetherian domain, then there exist just two possibilities for the prime ideal spectrum of $R[y]$, depending on whether or not $R$ is Henselian.
\end{abstract}

In this paper we investigate (i) the splitting of a prime ideal $P$ of a Noetherian domain $R$ in the absolute integral closure of $R(\S 1$ ), and (ii) the prime spectrum of $R[y]$, in case $R$ is also countable and semilocal of dimension one and $y$ is an indeterminate $(\S 2)$. The terminology $n$-split ideal, used by McAdam [M], for $P$ a prime ideal of a domain $A$ and $\bar{A}$ the integral closure of $A$ in an algebraic closure of the quotient field of $A$, means there are exactly $n$ primes in $\bar{A}$ (possibly $n=\infty$ ) which lie over $P$. Specifically for (ii), we are interested in what information about $R$ can be gleaned from $\operatorname{Spec} R[y]$ and which dimension 2 partially ordered sets arise as $\operatorname{Spec} R[y]$ for some $R$. Throughout we will be viewing $\operatorname{Spec} A$ as the partially ordered set of prime ideals of $A$, rather than as an affine scheme. When $A$ is Noetherian, the topology on the spectrum is uniquely determined by the partial ordering; so, in this case, viewing $\operatorname{Spec} A$ as a topological space is equivalent to viewing it as a partially ordered set.

As an example of this kind of information, we remark that if $V$ is a onedimensional local Noetherian domain with maximal ideal $\mathbf{m}$, then $V$ is Henselian if and only if each height one prime of the polynomial ring $V[y]$ other than $\mathbf{m} V[y]$ is contained in a unique maximal ideal. For by [ $\mathrm{N}, 43.12]$, $V$ is Henselian if and only if each domain extension of $V$ that is integral over

Received by the editors June 19, 1988 and, in revised form, September 26, 1988. The contents of this paper were presented to the American Mathematical Society at its eight hundred and forty fifth meeting, held at the University of Kansas in Lawrence on Saturday, October 29, 1988.

1980 Mathematics Subject Classification (1985 Revision). Primary 13A17, 13E05, 13 H99. 13J15; Secondary $13 \mathrm{C} 15$.

Key words and phrases. Prime ideal spectrum, polynomial ring, Noetherian ring, $n$-split ideal, Henselian ring.

Both authors were supported by the National Science Foundation. 
$V$ is quasilocal. This is easily seen to be equivalent to the fact that each domain extension which is integral over $V$ and is generated by a single element is quasilocal. Such extensions have the form $V[\bar{y}]=V[y] / P$. Hence if $V$ is not Henselian, then there is a height one prime of $V[y]$ that contains a monic polynomial in $y$ and is contained in more than one maximal ideal of $V[y]$. On the other hand, if $V$ is Henselian, then in view of the Krull-Akizuki Theorem [N, 33.2] it follows that if $P$ is any height one prime of $V[y]$ with $P \cap V=(0)$, then $V[y] / P$ is local, so $P$ is contained in a unique maximal ideal of $V[y]$. This shows that even for $V$ and $W$ countable discrete rank one valuation domains (so with order isomorphic two element prime spectra), Spec $V[y]$ and Spec $W[y]$ are not order isomorphic if $V$ is Henselian and $W$ is not.

These considerations were inspired by Roger Wiegand's paper [RW] in which he shows $\operatorname{Spec} \mathbb{Z}_{2}[x, y]$, polynomials in two variables over the 2 element field, is not order isomorphic to $\operatorname{Spec} \mathbb{Q}[x, y](\mathbb{Q}=$ the rationals); whereas of course $\operatorname{Spec} \mathbb{Z}_{2}[x]$ is order isomorphic to Spec $\mathbb{Q}[x]$. More precisely he proves for $R$ a two-dimensional domain that is finitely generated as an algebra over a field $k$, that $\operatorname{Spec} R \cong \operatorname{Spec} \mathbb{Z}[y]$ ( $\mathbb{Z}=$ the integers) if and only if $k$ is contained in the algebraic closure of a finite field.

Using a different approach, McAdam [M] proved two results which are relevant to this discussion: (for a Noetherian domain $R$ )

(1) Every nonzero prime ideal of the polynomial ring $R[y]$ is $\infty$-split.

(2) If $P$ is a height one prime which $n$-splits, every set of more than $n$ uppers to $P$ in $R[y]$ contains only one height one prime ideal, $P R[y]$, in its intersection, whereas the intersection of $n$ or fewer uppers of $P$ contains infinitely many primes of $R[y] ; n=\infty$ implies that the intersection of every finite set of uppers to $P$ contains infinitely many primes.

Thus a local rank one domain is Henselian if and only if its maximal ideal 1-splits. (This observation can also be deduced from Artin's paper [A, 1.4] and [N, 33.2].)

We show, in Theorem 1.1 of $\S 1$, that every prime ideal in a Noetherian domain $R$ is either 1 -split or $\infty$-split, that is, $n$ can only be 1 or $\infty$. Furthermore $R$ contains at most one nonzero 1 -split prime ideal and if such a prime exists it is the unique maximal ideal of $R$. This does not uniquely determine the prime spectrum of $R[y]$ in case $R$ is a one-dimensional Noetherian domain with $\infty$-split maximals, since, as mentioned above, $\mathrm{R}$. Wiegand proved that $\operatorname{Spec} \mathbb{Z}_{2}[x, y]$ is not order isomorphic to $\operatorname{Spec} \mathbb{Q}[x, y]$. Hence by taking $R_{1}=\mathbb{Z}_{2}[x]$ and $R_{2}=\mathbb{Q}[x]$ one can have $\operatorname{Spec} R_{1} \cong \operatorname{Spec} R_{2}$ and yet Spec $R_{1}[y] \not$ Spec $R_{2}[y]$. However, for a countable one-dimensional semilocal domain $R$, Spec $R[y]$ is either of the Henselian type or a more standard type (Theorem 2.7). An application of this is that localizing $\mathbb{Q}[x]$ at a finite number of primes before adjoining $y$ yields a spectrum order isomorphic to that 
obtained by localizing $\mathbb{Z}_{2}[x]$ (or $\mathbb{Z}$ ) at the same number of primes and then adjoining $y$.

\section{Splitting in a Noetherian domain}

In this section we demonstrate the following theorem:

1.1 Theorem. Suppose $R$ is a Noetherian domain.

(i) Every nonzero prime ideal $\mathbf{m}$ is either 1-split or $\infty$-split.

(ii) If $\mathbf{m}$ is a nonzero 1-split prime ideal of $R$, then $R$ is local with maximal ideal $\mathbf{m}$.

The proof requires two lemmas:

1.2 Lemma. If $S$ is an integrally closed domain with quotient field $F, P$ and $Q$ are distinct maximal ideals of $S$ and $Q^{2} \neq Q$, then there exists a finite separable algebraic field extension $L$ over $F$ such that there are at least two distinct prime ideals of the integral closure of $S$ in $L$ lying over $P$ in $S$.

Proof. Since $P$ and $Q^{2}$ are comaximal, $P Q^{2}=P \cap Q^{2}$ and $S / P Q^{2} \cong S / P \oplus$ $S / Q^{2}$ by the canonical map. Hence there exists a $z \in Q-Q^{2}$ such that $z \equiv$ $1 \bmod P$. If the characteristic of $S / P \neq 2$, consider $x^{2}-z \in S[x]$. If char $S / P=2$, consider $x^{3}-z$. Since $z \in Q-Q^{2}, x^{2}-z$ and $x^{3}-z$ have no root in $S$. But $S$ is integrally closed, so it follows that $x^{2}-z$ and $x^{3}-z$ are irreducible in $F[x]$.

If $\operatorname{char} S / P \neq 2$ and $\theta$ is a root of $x^{2}-z$, then

$$
S[\theta] / P S[\theta] \cong(S / P)[x] /\left(x^{2}-1\right) .
$$

Since $x^{2}-1=(x+1)(x-1)$ we see that in $S[\theta]$, there are two maximal ideals lying over $P$ in $S$ and the lemma follows with $L=F[\theta]$.

If char $S / P=2$, let $\tau$ be a root of $x^{3}-z$, then

$$
S[\tau] / P S[\tau] \cong(S / P)[x] /\left(x^{3}-1\right) .
$$

Since $x^{3}-1$ has at least 2 distinct factors over $S / P$, we see that there are at least 2 distinct maximal ideals of $S[\tau]$ lying over $P$ in $S$, so the lemma follows with $L=F[\tau]$.

1.3 Lemma. Suppose $P$ is a nonzero prime ideal in a Noetherian domain $R$. If $P$ is 1-split, then $P$ is comparable with every prime ideal $Q$ of $R$ (that is, $P \supseteq Q$ or $Q \supseteq P)$.

Proof. If not, let $Q$ be a prime ideal of $R$ not comparable to $P$. Without loss of generality $P$ and $Q$ are maximal (since $P$ is $n$-split exactly when $P(R-P-Q)^{-1} R$ is). Let $\bar{R}$ be the absolute integral closure of $R$-the integral closure in the algebraic closure of $F$, the quotient field of $R$-and let $S$ be the integral closure of $R$ in $F$. By [C], there exists a discrete rank one valuation ring $V$ between $R$ and $F$ with maximal ideal $\mathscr{N}$ such that $Q=\mathcal{N} \cap R$. It 
follows that $Q_{1}=\mathscr{N} \cap S$ is a maximal ideal of $S$ lying over $Q, \cap_{n=1}^{\infty} Q_{1}^{n}=(0)$ and so $Q_{1}^{2} \neq Q_{1}$. Thus if $P_{1}$ is a maximal ideal of $S$ lying over $P$, then by Lemma 1.2 there exists an integral extension $S_{1}$ of $S$, contained in $\bar{R}$, so that two distinct prime ideals of $S_{1}$ lie over $P_{1}$ and thus over $P$. Hence $P$ is not 1-split.

Proof of Theorem 1.1. For (i), suppose $\mathbf{m}$ is $n$-split, for $1<n<\infty$. Let $\bar{R}$ be the absolute integral closure of $R$; say $\mathbf{n}_{1}, \ldots, \mathbf{n}_{n}$ are the $n$ distinct prime ideals of $\bar{R}$ lying over $\mathbf{m}$. Choose $\theta \in \mathbf{n}_{1}-\bigcup_{i \neq 1} \mathbf{n}_{i}$ and $\beta \in \bigcap_{i \neq 1}\left(\mathbf{n}_{i}-\mathbf{n}_{1}\right)$. In $R_{1}=R[\beta, \theta], \mathrm{n}_{1} \cap R_{1}$ and $\mathrm{n}_{2} \cap R_{1}$ are not comparable; thus by Lemma 1.2, $\mathrm{n}_{1} \cap R_{1}$ is at least 2-split, but the only prime ideal of $\bar{R}$ lying over $\mathrm{n}_{1} \cap R_{1}$ is $\mathbf{n}_{1}$, a contradiction.

For (ii), if $\mathbf{m}$ is 1 -split but not the unique maximal ideal of $R$, then we choose a nonunit $a \in R-\mathbf{m}$. By the Krull principal ideal theorem [N, 9.2], there exists a height one prime ideal $\mathbf{n}$ containing $a$. It follows that $\mathbf{n}$ and $\mathbf{m}$ are not comparable, contradicting Lemma 1.3.

1.4 Proposition. For $R$ a Noetherian domain of dimension $n$, statements (i)(iv) are equivalent:

(i) $R$ contains a nonzero prime ideal $\mathbf{m}$ which is 1 -split.

(ii) Every domain extension of $R$ that is integral over $R$ is quasilocal.

(iii) $R$ is a local Henselian ring.

(iv) $R_{\mathbf{m}}$ is Henselian for a maximal ideal $\mathbf{m}$ of $R$.

Conditions (i)-(iv) imply (v) and, if $n=1$, (v) is equivalent to (i)-(iv).

(v) $R$ is local, $\mathbf{m}$ is the maximal ideal, and the intersection of every pair of height $n+1$ primes of $R[y]$ contains exactly one height $n$ prime ideal of $R[y]$, namely $\mathbf{m} R[y]$.

Proof. Suppose (i) holds; by Theorem 1.1 (ii), $\mathbf{m}$ is the only maximal ideal of $R$. Now the absolute integral closure $\bar{R}$ is quasilocal [N, 10.9] and (ii) holds. The fact that (ii) and (iii) are equivalent is [N, 43.12] and (iii) trivially implies (iv).

Also (iv) implies $\mathrm{m} R_{\mathrm{m}}$ is 1 -split (via the equivalence of (ii) and (iii) for $R_{\mathrm{m}}$ ) and thus $\mathbf{m}$ is 1 -split, so (i) holds. The equivalence of (iii) and (v) when the dimension is 1 was done in the second paragraph of the introduction to this paper. (Also, this follows from [M, Theorem 6].)

To show (i)-(iv) implies (v) for $n>1$, suppose $\mathscr{N}_{1}$ and $\mathscr{N}_{2}$ are prime ideals of $R[y]$ with height $n+1$. Then $\mathscr{N}_{1} \cap R=\mathbf{m}=\mathscr{N}_{2} \cap R$ and so $\mathscr{N}_{1} \cap \mathscr{N}_{2}$ contains $\mathbf{m} R[y]$. If $\mathscr{N}_{1} \cap \mathscr{N}_{2}$ contains a prime ideal $Q$ of $R[y]$ of height $n$, then $P=Q \cap R$ has height $n$ or $n-1$. But height $(P)=n$ only if $P=\mathbf{m}$ and $Q=\mathbf{m} R[y]$. On the other hand, if height $(P)=n-1$, then $R / P$ has dimension one and $\mathbf{m} / P$ is 1 -split. Therefore by the equivalence of $(\mathrm{v})$ and (i) for $n=1$, the only height one prime in $\mathscr{N}_{1} /(P R[y])$ and $\mathscr{N}_{2} /(P R[y])$ is $(\mathbf{m} R[y]) /(P R[y])$. Thus $Q /(P R[y])=(\mathbf{m} R[y]) / P R[y])$ so $Q=\mathbf{m} R[y]$ as desired. 
1.5 Remark. It seems likely to us that (v) of Proposition 1.4 does not imply (i)(iv) for $n>1$. This would follow, for example, for $n=2$, if there exists a twodimensional non-Henselian local domain $R$ such that $R / P$ is Henselian for each height one prime $P$ of $R$. It is easy to construct an $R$ that comes close to what we want here. For example, if $k$ is a field and $x$ and $z$ are indeterminates over $k$, then $R=k[[x]][z]_{(x, z)}$ is a two-dimensional non-Henselian regular local domain such that $R / P$ is Henselian for all but one of the height one primes $P$ of $R$, the one exception being $P=x R$.

1.6 Example. For $n$ any positive integer, there exists a (non-Noetherian) domain $R$ having a prime ideal $P$ such that $P$ is $n$-split. To construct such an example, let $K$ be any algebraically closed field and let $\overline{K(x)}$ denote the algebraic closure of the field $K(x)$ where $x$ is transcendental over $K$. There exist $n$ distinct rank one valuation domains $V_{1}, \ldots, V_{n}$ that contain $K$ and have quotient field $\overline{K(x)}$. For example, we could take $a_{1}, \ldots, a_{n}$ to be $n$ distinct elements of $K$ and $V_{i}$ to be an extension to $\overline{K(x)}$ of the valuation domain $K[x]_{\left(x-a_{i}\right)}$, for each $i$. Each $V_{i}$ has the form $V_{i}=K+\mathscr{M}_{i}$, where $\mathscr{M}_{i}$ is the maximal ideal of $V_{i}$. Let $P=\mathscr{M}_{1} \ldots \mathscr{M}_{n}$ and set $R=K+P$. Then $R$ is a one-dimensional quasilocal domain with maximal ideal $P$ and the integral closure of $R$ in $\overline{K(x)}$ is $V_{1} \cap \cdots \cap V_{n}$, a one-dimensional domain with precisely $n$ maximal ideals [N, 11.11]. It follows that $P$ is $n$-split.

1.7 Remark. It would be interesting to know whether for each positive integer $n$ there exists a normal domain $R$ that has a prime ideal $P$ which is $n$-split. Of course this is true for $n=1$, and it is also true for $n=2$. To see this, consider the algebraic closure $\overline{\mathbb{Q}}$ of the rational numbers inside the field $\mathbb{C}$ of complex numbers. Let $\phi$ be the restriction to $\overline{\mathbb{Q}}$ of the complex conjugate automorphism of $\mathbb{C}$ over $\mathbb{R}$. Then $\phi$ has fixed field $L=\overline{\mathbb{Q}} \cap \mathbb{R}$ and $[\bar{Q}: L]=2$. Let $V$ be an extension to $\overline{\mathbb{Q}}$ of the discrete rank one valuation domain $\mathbb{Z}_{(5)}$ on $\mathbb{Q}$. Since $\overline{\mathbb{Q}}=L[x] /\left(x^{2}+1\right)$ and $x^{2}+1$ has two distinct roots in $\mathbb{Z} / 5 \mathbb{Z}, \phi(V) \neq V$. Hence $V \cap L=R$ is a rank one valuation domain that has two extensions $V$ and $\phi(V)$ to $\overline{\mathbb{Q}}$. Therefore, if $P$ is the maximal ideal of $R$, then $P$ is 2-split.

\section{ReleVANT PARTIALly ORDERED SETS}

It is convenient to set some notation for discussing partially ordered sets: for $u$ an element and $T$ a subset of a partially ordered set $U$ of finite dimension, let $G(u)=\{v \in U \mid v>u\}, L(T)=\{x \in U \mid G(x)=T\}$. Let $\mathscr{M}(U)$ denote the set of maximal elements of $U$ of maximal height and let $\mathscr{M}_{i}(U)$ be the maximal elements of height $i$, for each $i$. We're interested in three particular two-dimensional partially ordered sets, named for rings which have these prime spectra. The first, from $[R W]$ is shown there to describe $\operatorname{Spec} \mathbb{Z}[y]$. 
2.1 Definition. A partially ordered set $U$ is called countable integer polynomial, or $C \mathbb{Z} P$, provided

(P0) $U$ is countable.

(P1) $U$ has a unique minimal element $u_{0}$.

(P2) $U$ has dimension 2 .

(P3) For each element $u$ of height one, $G(u)$ is (countably) infinite.

(P4) For each pair $u, v$ of distinct elements of height $1, G(u) \cap G(v)$ is finite.

(P5) Given a finite set $S$ of height one elements and a finite set $T$ in $\mathscr{M}(U)$, there is a height one element $w$ such that $G(w) \supseteq T \supseteq G(w) \cap G(s)$, for all $s \in S$.

We will show $\operatorname{Spec}\left(\mathbb{Z}-\bigcup_{i=1}^{n} \mathbf{p}_{i}\right)^{-1} \mathbb{Z}[y]$, where $\left\{\mathbf{p}_{i} \mid 1 \leq i \leq n\right\}$ is a finite set of primes, is of the following type:

2.2 Definition. A partially ordered set $U$ is countable $n$-localized integer polynomial or $C \mathbb{Z}(n) P$ provided (P0)-(P2) above, hold and:

(P3) There exist infinitely many height one maximal elements.

(P4) There exist $n$ height one nonmaximal elements $u_{1}, \ldots, u_{n}$ (called special elements) satisfying: (i) $G\left(u_{1}\right) \cup \cdots \cup G\left(u_{n}\right)=\mathscr{M}(U)$; (ii) $G\left(u_{i}\right) \cap G\left(u_{j}\right)=\phi$ for $i \neq j$; (iii) $G\left(u_{i}\right)$ is infinite for each $i$, $1 \leq i \leq n$.

(P5) For each height one, nonmaximal, nonspecial element $u, G(u)$ is finite (and nonempty).

(P6) For each nonempty finite subset $T$ of $\mathscr{M}(U), L(T)$ is infinite.

Pictorially, a $C \mathbb{Z}(n) P$ partially ordered set looks like this:

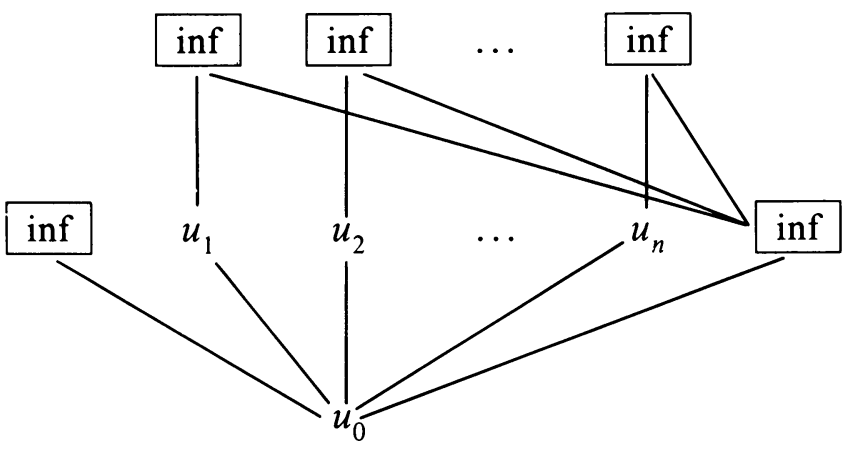

(The relationships of the lower right boxed section, determined by (P5) and (P6), are too complicated to display.)

Note that if $V$ is a Henselian discrete rank one valuation domain then (P6) of $C \mathbb{Z}(1) P$ is not satisfied for Spec $V[y]$, since the introductory remarks imply that whenever $T$ is bigger than a singleton set, $L(T)=\phi$. (For $R=\mathbb{Z}_{(2)}[y],(\mathrm{P} 6)$ holds; for example with $T=\{(2, y),(2, y+1)\}, L(T)$ contains $(y(y+1)+2)$, 
$(y(y+1)+4)$ and so on.) We are inspired to define a third partially ordered set:

2.3 Definition. A partially ordered set is countable Henselian polynomial, $C H P$, provided:

(P0)-(P5) of $C \mathbb{Z}(1) P$ hold, although (P5) could be sharpened to say " $G(u)$ is a singleton set".

(P6) For $T$ a finite subset of $\mathscr{M}(U)$ of cardinality greater than one, $L(T)=$ $\phi$; for $T$ a singleton set, $L(T)$ is infinite.

Picture:

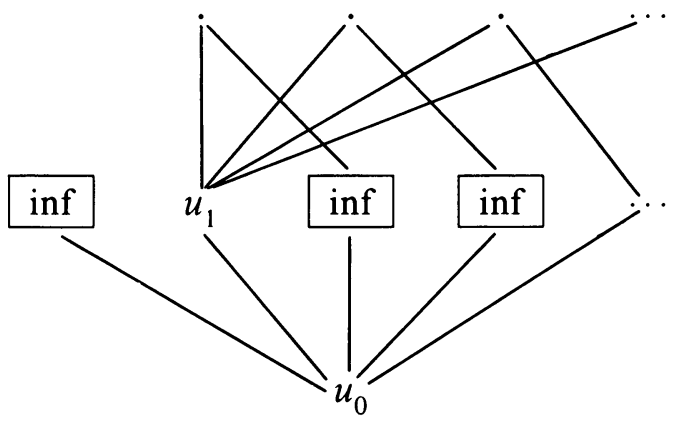

2.4 Definition. For a $C \mathbb{Z} P$ partially ordered set $U$ and $u_{1}, \ldots, u_{n}$ particular height one elements which are pairwise comaximal (that is, $G\left(u_{i}\right) \cap G\left(u_{j}\right)=\phi$ for $i \neq j)$, define $U$ localized at $G\left(u_{1}, \ldots, u_{n}\right)$, written $U\left(u_{1}, \ldots, u_{n}\right)$, to be the partially ordered subset $(U-\mathscr{M}(U)) \cup G\left(u_{1}\right) \cup \cdots \cup G\left(u_{n}\right)$.

2.5 Proposition. If $U$ is a $C \mathbb{Z} P$ partially ordered set, $n$ is a positive integer, and $u_{1}, \ldots, u_{n}$ are $n$ pairwise comaximal height one elements, then $U\left(u_{1}, \ldots, u_{n}\right)$ is a $C \mathbb{Z}(n) P$ partially ordered set.

Proof. Clearly $U\left(u_{1}, \ldots, u_{n}\right)$ satisfies (P0)-(P2), (P4) of $C \mathbb{Z}(n) P$. For (P5), suppose $u$ were a height one nonmaximal, nonspecial element of $U\left(u_{1}, \ldots, u_{n}\right)$ and $G(u)$ (in $U$ localized) were infinite. Then $G(u) \cap G\left(u_{i}\right)$ would be infinite for some $i$ and so in $U, G(u) \cap G\left(u_{i}\right)$ would be infinite, contradicting (P4) of $C \mathbb{Z} P$. For (P3), suppose the set of height one maximal elements were finite. Let

$$
S=\left\{\text { height one maximals of } U\left(u_{1}, \ldots, u_{n}\right)\right\} \cup\left\{u_{1}, \ldots, u_{n}\right\},
$$

and let $T=\phi$. By (P5) of $C \mathbb{Z P}$, there exists a height one $w \in U$ with $G(w) \cap G(s)=\phi$, for each $s \in S$. Then $G(w) \cap G\left(u_{i}\right)=\phi$, so $w \in$ $\mathscr{M}_{1}\left(U\left(u_{1}, \ldots, u_{n}\right)\right)$, and $w \in S$; now $G(w)=G(w) \cap G(w)=\phi$ (in $U$ ) is impossible. Thus $S$ is infinite, and (P3) holds. The proof of (P6) of $C \mathbb{Z}(n) P$ is similar: for $T$ a nonempty finite subset of $\mathscr{M}_{2}\left(U\left(u_{1}, \ldots, u_{n}\right)\right)$, the assumption that $S=L(T) \cup\left\{u_{1}, \ldots, u_{n}\right\}$ is finite leads to a contradiction of (P5) of $C \mathbb{Z} P$. 
2.6 Proposition. (1) If $U$ and $V$ are both $C \mathbb{Z}(n) P$ partially ordered sets, for some positive integer $n$, then $U$ is order isomorphic to $V$.

(2) If $U$ and $V$ are both $C H P$, then $U \cong V$.

Proof. Actually, part (1) follows from [RW, Theorem 1], but for completeness we outline a proof of both parts together, by defining a bijection $f$ from $U$ to $V$. Let $f$ take the minimal element of $U$ to that of $V$, the height 1 maximal elements of $U$ to those of $V$ and the special elements of $U$ to those of $V$ (exactly how not being important). If $u$ is a special element of $U$, let $f$ take the elements in $G(u)$ to the elements in $G(f(u)$ ) (exactly how not being important). It remains to define $f$ on the height 1 nonmaximal nonspecial elements of $U$. Let $x_{1}, x_{2}, x_{3}, \ldots$ and $y_{1}, y_{2}, y_{3} \ldots$ be the height 1 nonspecial nonmaximal elements of $U$ and $V$ respectively. Suppose that $f$ has already been defined on $x_{1}, x_{2}, \ldots, x_{i-1}$. Let $G\left(x_{i}\right)=\left\{z_{1}, \ldots, z_{m}\right\}$. Since $L\left(\left\{f\left(z_{1}\right), \ldots, f\left(z_{m}\right)\right\}\right.$ is infinite, let $j$ be the least integer such that $y_{j}$ is in $L\left(\left\{f\left(z_{1}\right), \ldots, f\left(z_{m}\right)\right\}\right)$ but not in $\left\{f\left(x_{1}\right), \ldots, f\left(x_{i-1}\right)\right\}$. Define $f\left(x_{i}\right)=$ $y_{j}$. It is not hard to see that $f$ is onto, and is an order isomorphism from $U$ to $V$.

The following proof is adapted from [M, Theorem 3]:

2.7 Theorem. Suppose that $n$ is a positive integer. Let $R$ be a countable Noetherian domain of dimension one with exactly $n$ maximal ideals. Then:

(i) If $n=1$ and the maximal ideal of $R$ is 1-split, $\operatorname{Spec} R[y]$ is $C H P$.

(ii) Otherwise (that is, $n \neq 1$ or $n=1$ but the maximal ideal is not 1-split), $\operatorname{Spec} R[y]$ is $C \mathbb{Z}(n) P$.

Proof. Let $K$ be the quotient field of $R, \mathbf{p}_{1}, \ldots, \mathbf{p}_{n}$ the maximal ideals and for each $i, k_{i}=R / \mathbf{p}_{i}$, the residue field. For (P3), let $0 \neq \beta \in \bigcap_{i=1}^{n} \mathbf{p}_{i}$, $\alpha \in R-\bigcup_{i=1}^{n} \mathbf{p}_{i}$, then $\left(\beta^{j} y+\alpha\right) K[y] \cap R[y]$ is a maximal height one prime ideal of $R[y]$ for each positive integer $j$. For (P4), let each $u_{i}$ be $\mathbf{p}_{i} R[y]$. If $\mathscr{M}$ is a maximal ideal of height 2 then $\mathscr{M} \cap R=\mathbf{p}_{i}$ for some $i$. Thus $\mathscr{M}$ is in $G\left(u_{i}\right)$. Also $G\left(u_{i}\right)$ corresponds to the maximal ideals of $k_{i}[y]$; thus $G\left(u_{i}\right)$ is infinite. For (P5), note that if $u$ is a nonspecial height one, $G(u)$ is a finite union of all the $G(u) \cap G\left(u_{i}\right)$ which are finite since $R[y]$ is Noetherian.

Now let $T$ be a nonempty finite subset of $\mathscr{M}_{2}(\operatorname{Spec} R[y])$. For (i), if $|T|>1$, Proposition $1.4(\mathrm{v})$ applies and $L(T)=\phi$. If $|T|=1$, say $T=\{\mathbf{n}\}$; since $R[y]$ is Noetherian, there are infinitely many primes between $(0)$ and $\mathbf{n}$. However, by $1.4(\mathrm{v})$, each (except $\mathrm{m} R[y]$ ) is contained in exactly one height two prime, namely $\mathbf{n}$. Thus $L(T)$ is infinite.

For (ii), suppose

$$
T=\left\{M_{11}, \ldots, M_{1 t_{1}}, M_{21}, \ldots, M_{2 t_{2}}, \ldots, M_{r 1}, \ldots, M_{r t_{r}}\right\},
$$

where we assume $1 \leq r \leq n$ and $M_{i j} \in G\left(u_{i}\right)$ for all $1 \leq i \leq r, 1 \leq j \leq t_{i}$. Then for each $i, j, M_{i j}=\left(\mathbf{p}_{i}, g_{i j}\right)$ for some monic polynomial $g_{i j}$ of $R[y]$ which is irreducible mod $\mathbf{p}_{i}$. Now by Theorem 1.1, the $\mathbf{p}_{i}$ are $\infty$-split, and 
so there exists a finitely generated integral extension $R^{\prime}$ of $R$ so that each $\mathbf{p}_{i}$ has at least $t_{i}$ distinct primes of $R^{\prime}$ lying over it. Then $R^{\prime}$ is a semilocal Noetherian one-dimensional domain. Let $\mathbf{p}_{i 1}^{\prime}, \ldots, \mathbf{p}_{i t_{i}}^{\prime}$, denote $t_{i}$ primes lying over $\mathbf{p}_{i}$, for each $i$. In $R^{\prime}[y]$ choose height two maximal ideals $M_{i j}^{\prime}$ lying over the elements of $T$ and the $\mathbf{p}_{i j}^{\prime}$ : that is, for each $1 \leq i \leq r$ and $1 \leq j \leq t_{i}$, $M_{i j}^{\prime} \cap R[y]=M_{i j}, M_{i j}^{\prime} \cap R^{\prime}=\mathbf{p}_{i j}^{\prime}, M_{i j}^{\prime}=\left(\mathbf{p}_{i j}^{\prime}, g_{i j}^{\prime}\right)$, where $g_{i j}^{\prime}$ is monic and irreducible $\bmod \mathbf{p}_{i j}^{\prime}$. (The effect of this maneuver is that each prime ideal of the new coefficient ring is contained in at most one of the maximal ideals under consideration in the new polynomial ring.) Also as in the proof of [M, Theorem 1], there exists a finitely generated integral extension $S$ of $R^{\prime}$ so that if $1 \leq i \leq r, 1 \leq j \leq t_{i}$, we can choose $\mathbf{q}_{i j}$ in $S$ lying over $\mathbf{p}_{i j}^{\prime}, N_{i j}$ in $S[y]$ lying over $M_{i j}^{\prime}$ with $N_{i j}=\left(\mathbf{q}_{i j}, y+a_{i j}\right)$ for some $a_{i j} \in S$.

We now work in $S$, a finitely generated integral extension of $R$. (Hence $S$ is a semilocal one-dimensional domain and its quotient field $L$ is a finite extension of $K$.) For convenience we relabel the nonzero prime ideals of $S$ as $\mathbf{q}_{1}, \ldots, \mathbf{q}_{n}$. Also we consider $T^{\prime}=\left\{\mathscr{N}_{1}, \ldots, \mathscr{N}_{r}\right\}$ to be the subset of $\mathscr{M}(\operatorname{Spec} S[y])$ chosen to intersect down to the original $T$ in $\mathscr{M}(\operatorname{Spec} R[y])$; we have $\mathscr{N}_{i}=\left(\mathbf{q}_{i}, y+a_{i}\right)$, for $a_{i} \in S$. Use the Chinese Remainder Theorem to find $d, c$, and $g$ in $S$ such that

(i) For $1 \leq i \leq r, d \equiv 1, c \equiv a_{i}, g \equiv 0 \bmod \mathbf{q}_{i}$.

(ii) For $r<i \leq n, d \equiv 0, c \equiv 0, g \equiv 1 \bmod \mathbf{q}_{i}$.

By [M, Lemma 2], $Q_{j}=\left(y+\left(c+g^{j}\right) / d\right) L[y] \cap S[y]$ is the unique minimal prime divisor of $\left(d y+c+g^{j}\right) S[y]$ since $d$ and $c+g^{j}$ are relatively prime in $S$. Certainly $G\left(Q_{j}\right) \supseteq T^{\prime}$, for each positive integer $j$, relative to $\operatorname{Spec} S[y]$. Also if $d y+\left(c+g^{j}\right) \in \mathscr{N}$, a maximal ideal of $S[y]$, then $\mathscr{N} \supseteq \mathbf{q}_{i}$, for some $i$, $1 \leq i \leq n$. If $1 \leq i \leq r$, then $\mathscr{N} \supseteq\left(\mathbf{q}_{i}, y+a_{i}\right)$, so $\mathscr{N}=\mathscr{N}_{i}$. If $r<i \leq n$, then $d y+\left(c+g^{j}\right) \equiv 1 \bmod \mathbf{q}_{i}$, so $\mathscr{N}=S[y]$, a contradiction. Thus $T^{\prime}=G\left(Q_{j}\right)$ and so $Q_{j} \in L\left(T^{\prime}\right)$; hence $L\left(T^{\prime}\right)$ is infinite.

Finally, in order to complete the argument for (iii), we return to $T$ and $R[y]$ : there are infinitely many distinct prime ideals $I_{j}=Q_{j} \cap R[y]$ since the extension $S$ was finitely generated over $R$. Also each $I_{j}$ is contained in each member of $T$; that is $G\left(I_{j}\right) \supseteq T$. Now if $\mathscr{N} \supseteq I_{j}$, for some maximal ideal $\mathscr{N}$ of $R[y]$, then by Going Up [N, 10.9], there exists a maximal ideal $\mathscr{N}^{\prime}$ of $S[y]$ lying over $\mathscr{N}$ with $\mathscr{N}^{\prime} \supseteq Q_{j}$. But $G\left(Q_{j}\right)=T^{\prime}$ and so $\mathscr{N}^{\prime} \in T^{\prime}$. Hence $\mathscr{N}=\mathscr{N}^{\prime} \cap R[y] \in T$; that is, $T \supseteq G\left(I_{j}\right)$. Thus each $I_{j}$ is an element of $L(T)$ and so $L(T)$ is infinite.

2.8 Corollary. (1) For $\mathbf{p}_{1}, \ldots, \mathbf{p}_{n}$ nonzero prime ideals of $\mathbb{Z}$ and

$$
L=\left(\mathbb{Z}-\bigcup_{i=1}^{n} \mathbf{p}_{i}\right)^{-1} \mathbb{Z},
$$

$\operatorname{Spec} L[y]$ is $C \mathbb{Z}(n) P$. 
(2) For $k$ a countable field, $x$ and $y$ indeterminates, $\mathbf{q}_{1}, \ldots, \mathbf{q}_{n}$ maximal ideals of $k[x]$ and $R=\left(k[x]-\bigcup_{i=1}^{n} \mathbf{q}_{i}\right)^{-1} k[x]$, Spec $R[y]$ is $C \mathbb{Z}(n) P$.

(3) For $L$ and $R$ as above, $\operatorname{Spec} L[y] \cong \operatorname{Spec} R[y]$.

Proof. This follows from [RW, Theorem 2], 2.6 and 2.7.

Added in proof. In joint work with S. Abhyankar, the authors have produced the example needed in Remark 1.5, to show (v) does not imply (i)-(iv) of 1.4. This work is a study of power series rings and will be submitted for publication soon.

\section{ACKNOWLEDGMENT}

We wish to thank Brian Harbourne, Craig Huneke, Larry Levy, Stephen McAdam, Roger Wiegand and the referee for helpful comments regarding this paper.

\section{REFERENCES}

[A] M. Artin, On the joins of Hensel rings, Adv. in Math. 7 (1971), 282-296.

[C] C. Chevalley, La notion d'anneau de décomposition, Nagoya Math. J. 7 (1954), 21-33.

[K] I. Kaplansky, Commutative Rings, Boston, Allyn \& Bacon, 1970.

[M] S. McAdam, Intersections of height two primes, J. Algebra 49 (1977), 315-321.

[N] M. Nagata, Local Rings, Interscience, New York, 1962.

[RW] R. Wiegand, The prime spectrum of a two-dimensional affine domain, J. Pure \& Appl. Algebra 40 (1986), 209-214.

[RW'] _ Homeomorphisms of affine surfaces over a finite field, J. London Math. Soc. (2) 18 (1978), 28-32.

Department of Mathematics, Purdue University, West Lafayette, Indiana 47907

Department of Mathematics \& Statistics, University of Nebraska-Lincoln, Lincoln, NEBRASKA 68588-0323 